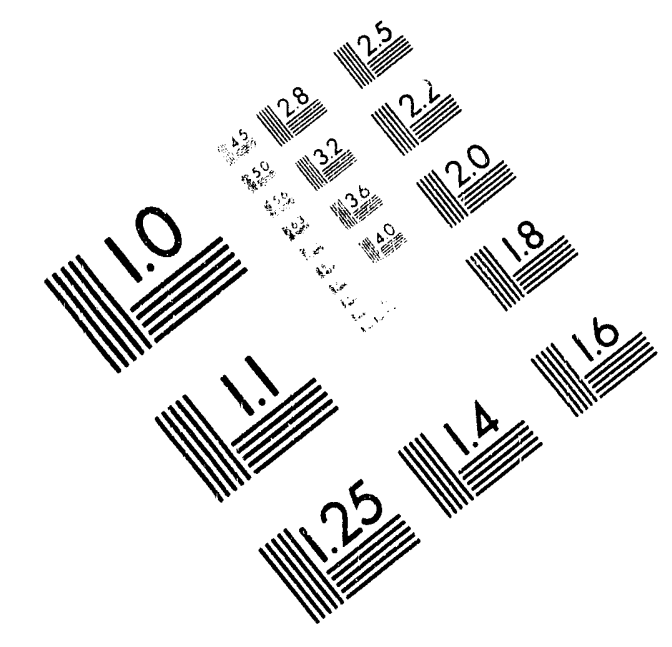

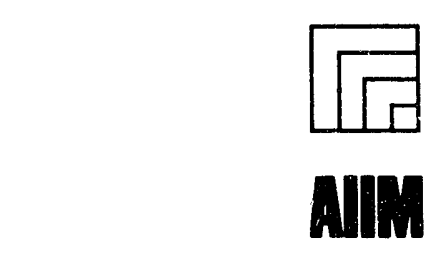

Association for Information and Image Management

1100 Wayne Avenue, Suite 1100

Silver Spring. Maryland 20910

301/587-8202

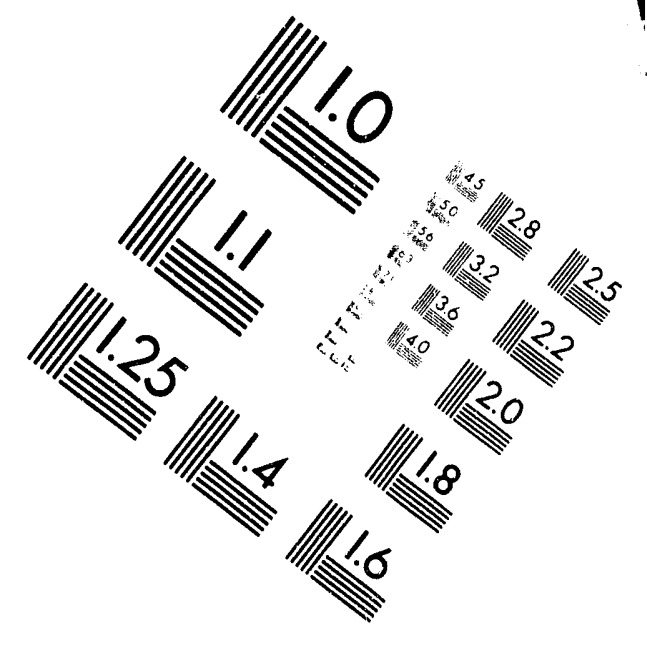

\title{
Centimeter
}

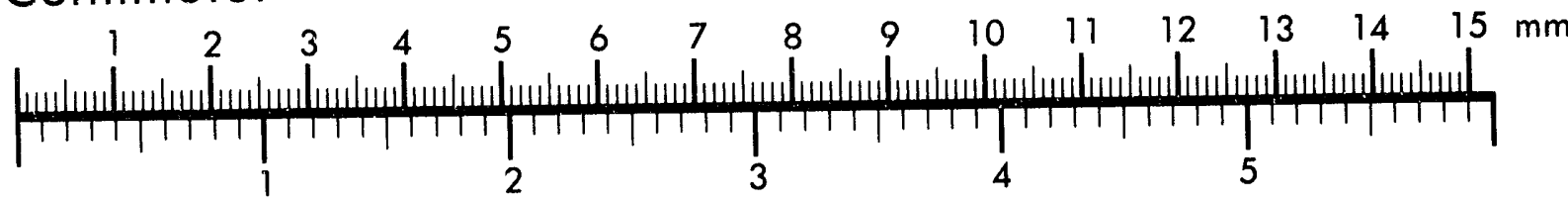

Inches
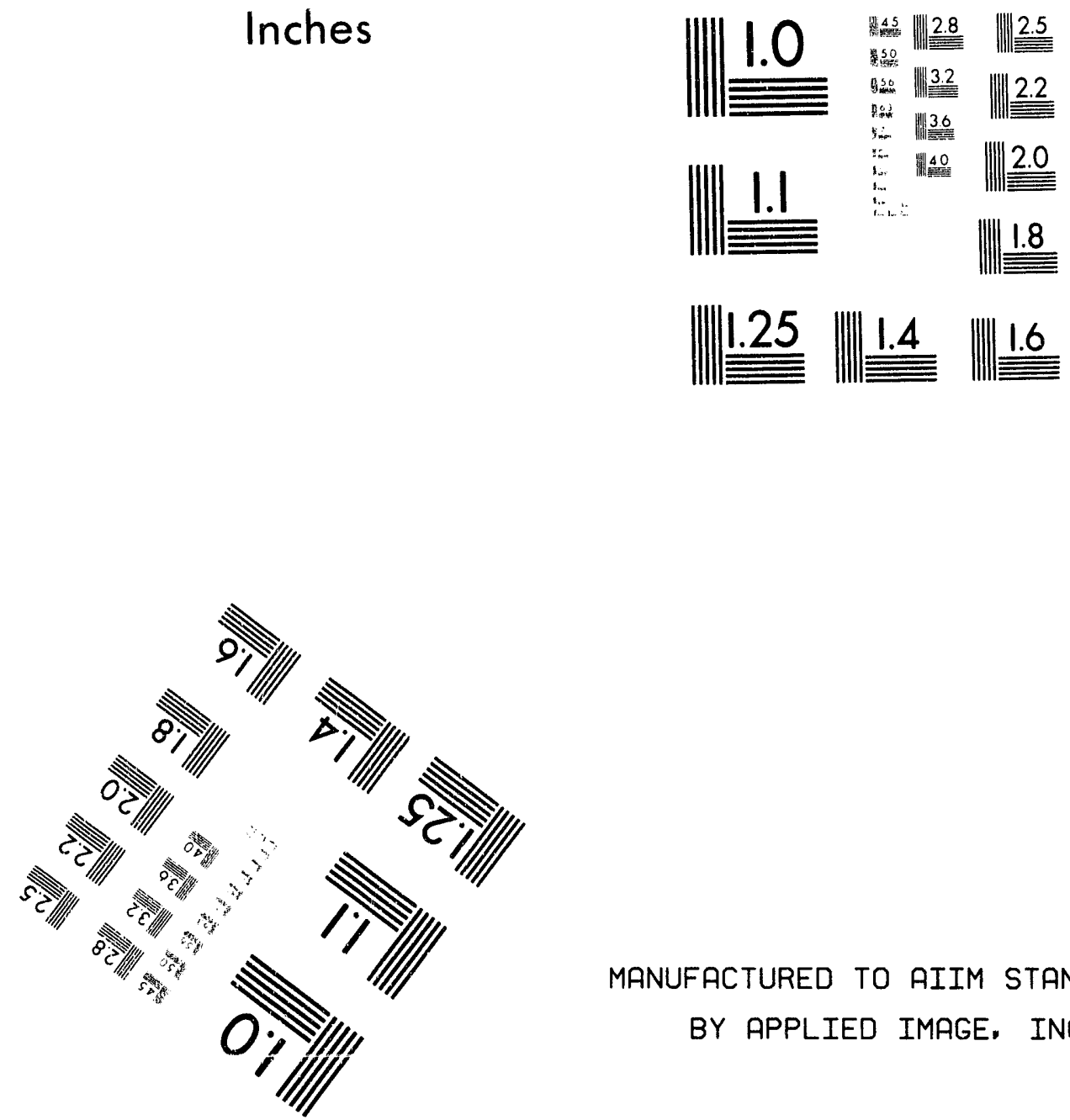

MANUFACTURED TO AIIM STANDARDS

BY APPLIED IMAGE, INC.

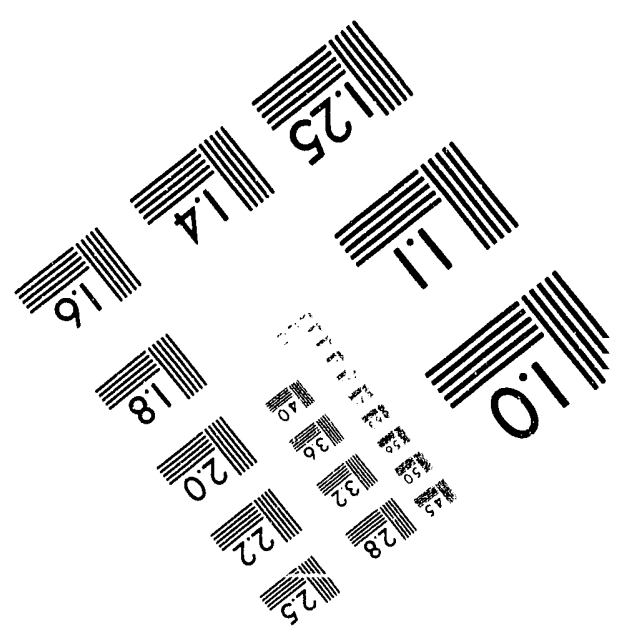



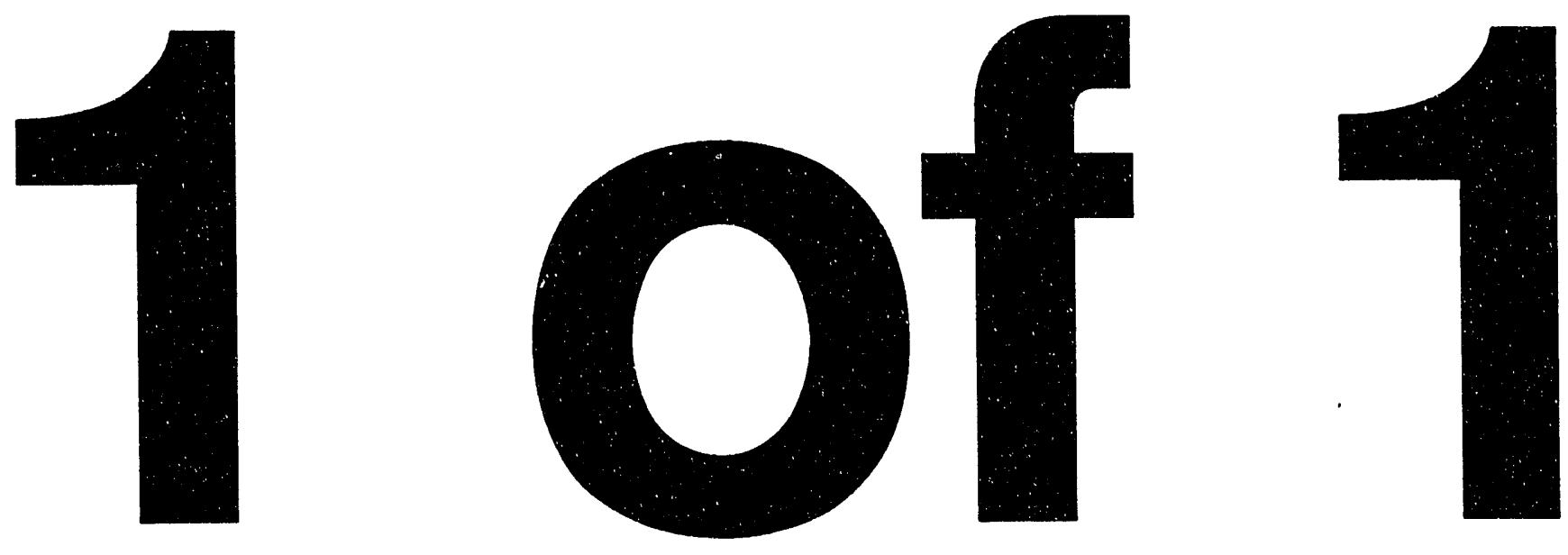


\title{
Zuf-9309116..
}

\section{Vibration Suppression by Modulation of Elastic Modulus Using Shape Memory Alloy 1}

\author{
Daniel J. Segalman \\ Department 1434 \\ Sandia National Laboratories \\ Gordon G. Parker \\ Department of Mechanical and Aerospace Engineering \\ State University of New York \\ at Buffalo \\ D. J. Inman \\ Department of Engineering Science and Mechanics \\ Virginia Polytechnic and State University \\ Blacksburg, VA
}

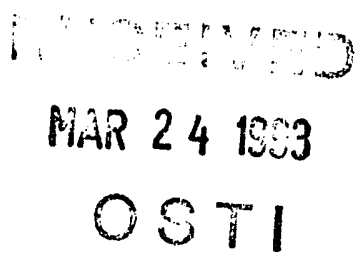

\subsection{Introduction}

Most common applications of "smart materials" actuators involves obliging them to undergo some generalized displacement in response to a specified stimulus. A slightly different approach is suggested in this paper. Here, we consider an application in which a modulus rather than a displacement is manipulated. Further, we present a class of problems for which such a smart material can be used to address very simply a problem of rotating equipment.

The first portion of this paper proposes a method of fabricating a material whose modulus can be changed substantially through the application of a specified stimulus. The particular implementation presented here indirectly exploits the large deformation associated with shape memory alloys to achieve the desired modulation of stiffness.

The next portion of this paper discusses a class of vibration problems for which such materials have a serious potential for vibration suppression. These are problems, such as the spinning up of rotating machinery, in which the excitation at any time lies within a nar. row frequency band, and that band moves through the frequency spectrum in a predictable manner.

Finally, an example problem is examined and the utility of this approach is discussed.

1. Work supported by the U.S. Department of Energy at Sandia National Laboratories under Contract DEAC0476DP00789

aLSTRIBUTION OF THIS DUCUMENT IS UNLIMITEN 


\subsection{A Smart Material with Modulated Elastic Modulus}

Here we present an approach for modulating the elastic moduli of materials by introducing a reinforcing material whose contribution to the stiffness of the composite can be turned on and off. We first consider an elastic matrix structure through which an array of holes has been drilled. Then strands of shape memory alloy (SMA) are threaded through the holes and knobs (or knots) are placed at the ends of the strands, leaving just a little slack. (See Figure 1 and Figure 2.) Since the SMA can move freely through the matrix, it contributes nothing to the stiffness of the composite structure.

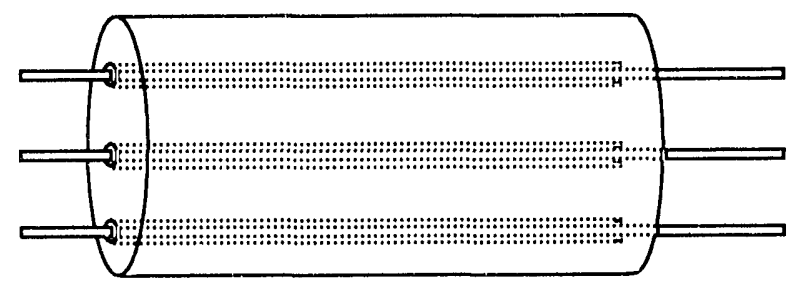

FIGURE 1. The matrix material with holes through which shape memory alloy (SMA) is threaded. The free movement of the SMA with respect to the matrix prevents it from contributing to the stiffness of the composite structure.

We now take advantage of a fundamental feature of shape memory alloy: when shape memory alloy is subjected to a change of temperature it is caused to jump to an equilibrium phase appropriate to that temperature. In particular, it can be switched from the extended, stress-free state shown in Figure 2 to the shortened state shown in Figure 3. In the shortened state, all slack is taken up, the knobs are pulled into the matrix, and the SMA is in tension.

This state, in which the SMA is contracted, the knobs fully couple the extensional deformation of the SMA to that of the matrix material. One may now use any one of the standard formulae to estimate the extensional modulus of the composite structure. A good 


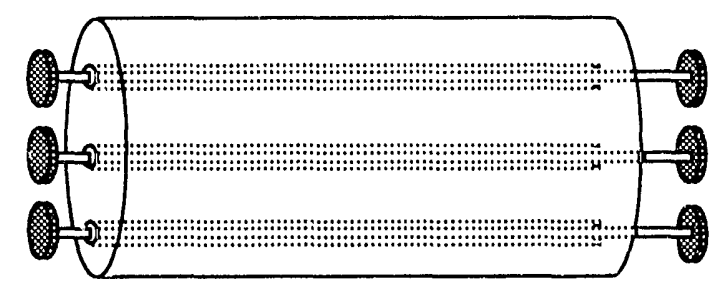

FI(jURF 2. The matrix material with holes through which shape memory alloy (SMA) is threaded. Even though there are knobs on the ends of the SMA strands, there is still enough slack to permit free movement. Again, the free movement of the SMA with respect to the matrix prevents it from contributing to the stiffiness of the composite structure

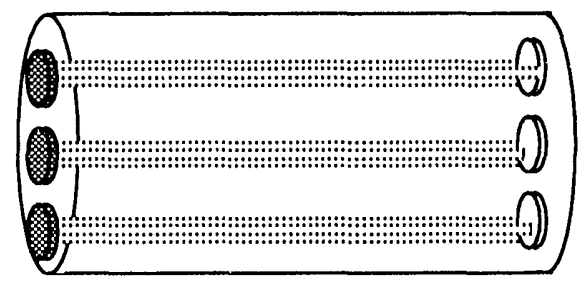

FIGURE 3. The matrix material with holes through which shape memory alloy (SMA) is threaded. The SMA has been activated, causing a junp to a phase with a shorter equilibrium length, taking up all slack. The knobs are now pressing against the matrix material, completely coupling extensional deformations of the two naterials.

review of alternative methods of performing these calculations is presented in Reference 1. A first approximation of the stiffening effect of the SMA is obtained by assuming uniform strains within the composite. This analysis results in a stiffness increase proportional to the relative stiffness of the SMA and the matrix material, to the ratios of the cross sectional areas of the SMA and the matrix materials, and to the percentage of the SMA strands that have been activated to contract.

The phase transformations of the SMA is reversible, so that a return to the previous temperature will result in a return to the configuration shown in Figure 2. By raising and dropping temperature, on can cause repeated reversals in the stiffness of the composite. The issue of response time becomes primarily an issue of the rates of heating and cooling. Heating is usualiy achieved by running a current through the SMA itself while cooling is usually achieved through convective and diffusive processes. However, response times can be accelerated by using more aggressive cooling techniques Reference 2 . 
It is important to note that the effect that is being targeted here is to modulate stiffness rather than to impose a deformation, as has been done often before; see Reference 4 . A. Baz et al in Reference 3 have used shape-memory alloy embedded in a composite to achieve a more gradual modulation in stiffness, though the physical mechanism is still a point of controversy.

Because applications described below require a change in modulus just from one stage of a transient spinning process to another, rather than within each rotation, particularly fast response times are not necessary.

It should be noted that the strategy presented above for modulating the stiffness of a composite structure could be applied with materials other than shape memory alloy, so long as those materials can be caused to undergo large static deformation through some outside stimulus. Classes of such materials besides shape memory alloys include thermo-elastic, piezo-electric, and magnetostrictive materials.

A further note should observe that the crude configuration shown above could be extended by placing the SMA near the surface of a beam, and staggered along the length of the beam. This configuration, shown in Figure 4, permits effective modulation of the bending modulus of the beam. If the beam is originally in a spiral configuration, one now has a spring whose rate is modulated.

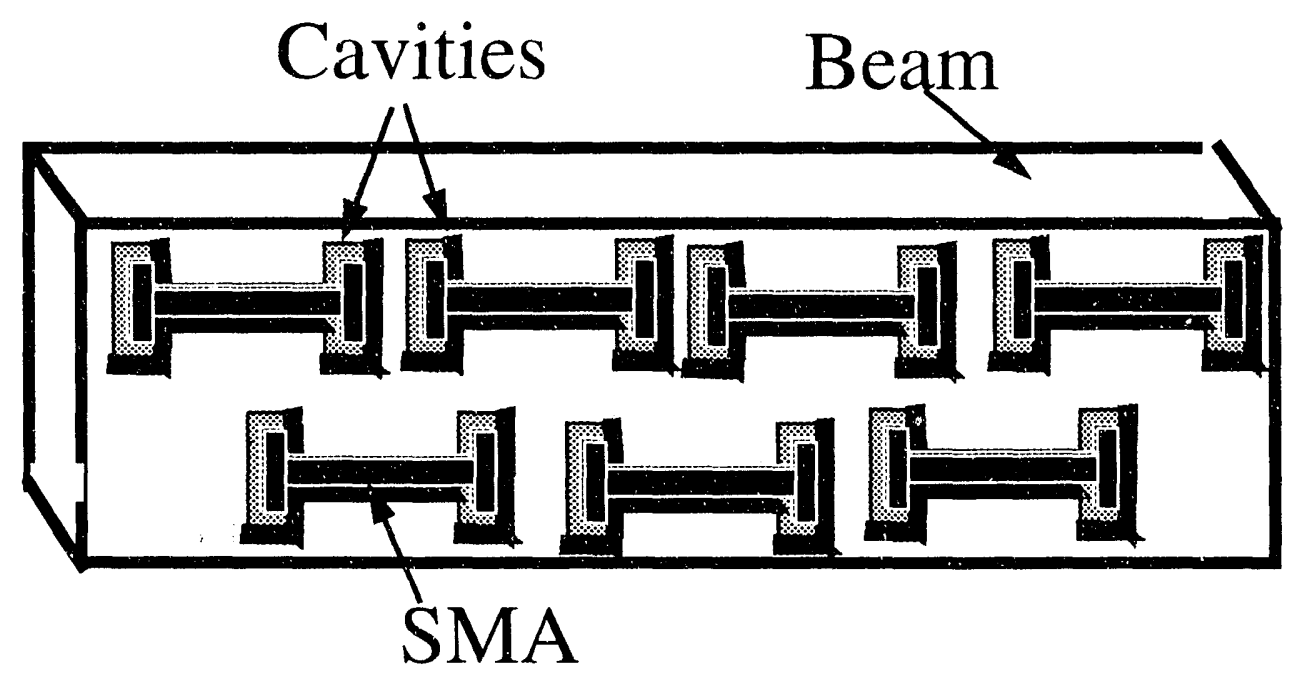

FIGURE 4. A staggered configuration of shape memory alloy fillers in an elastic matrix. 


\subsection{Application to a Class of Vibration Problems}

\section{General Problem Description}

The vibration suppression capabilities of the smart material described above will be demonstrated by application to a class of rotating vibration problems. This class of problems is defined by the excitation frequency of the system being an integer multiple, $n$, of the angular velocity of the system so that during spin-up, the excitation frequency passes through the natural frequency of the system.

\section{Paradigm Problem Description}

We consider here the simplest such case, that of a rigid disk pinned to a rigid shaft. The connection includes a keyway so that the disk must rotate with the shaft, though it may wobble from side to side. A torsional spring serves to restore the disk to its normal configuration. This simple model, used in other dynamics analysis in Reference 5, is meant to account in a very approximate way for shaft or disk flexibility. The disk rotates between frictionless spring loaded plates. A number, $n$, of small sinusoidally shaped projections on the surface of the disk cause a sinusoidal excitation. The torsional spring is oriented such that a line connecting the peaks of the projections on the disk would be perpendicular to the axis of rotation of the torsional spring. This system is shown in Figure 5.

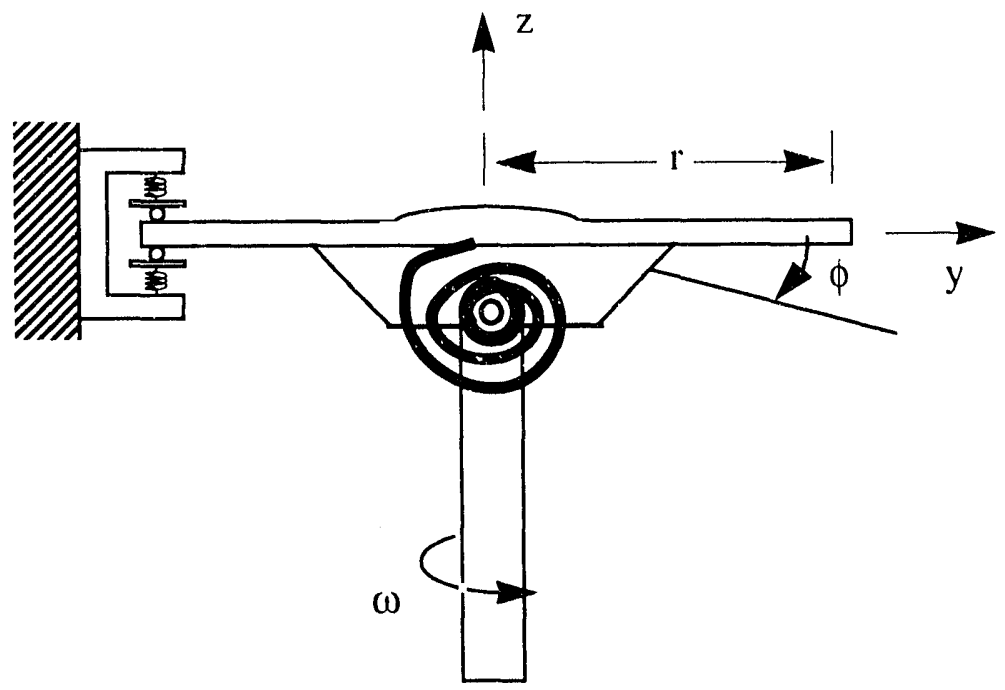

FICURE 5. I)isk/Rotor System

The governing equations can be derived in many ways (the authors have used the method presented in Reference 6). The equation for the angle $\phi$, by which the disk is off normal is: 


$$
{ }_{y y} \ddot{\phi}+c \dot{\phi}+\phi\left(\beta k+I_{y y} \omega^{2}\right)=A \sin (n \omega t)
$$

The multiplier $\beta$ is ordinarily unity, but will be used later on as a "stiffening" multiplier. An interesting feature of this system is that the excitation frequency at resonance is:

$$
\omega^{2}=\frac{\frac{k}{I_{y y}}-\left(\frac{c}{2 I_{y y}}\right)^{2}}{n^{2}-1}
$$

so that excitation will pass through the damped natural frequency of the system only for $n$ greater than one.

The following quantities are used to make the equation of motion dimensionless:

$$
\begin{array}{ll}
\omega_{o}=\sqrt{\frac{k_{o}}{I_{y y}}} & \text { natural frequency for the nonspinning system. } \\
\alpha=\frac{\omega}{\omega_{o}} & \text { dimensionless frequency } \\
T=\omega_{o} t & \text { dimensionless time } \\
\xi=\frac{c}{2 I_{y y} \omega_{o}} & \text { dimensionless damping ratio } \\
\tilde{A}=\frac{A}{\omega_{o}^{2} I} & \text { dimensionless excitation amplitude } \\
\Phi(T)=\phi\left(\frac{t}{\omega_{o}}\right) & \text { dimensionless deflection amplitude }
\end{array}
$$

The dimensionless equation of motion is:

$$
\Phi^{\prime \prime}+2 \xi \Phi^{\prime}+\left(\beta+\alpha^{2}\right) \Phi=\tilde{A} \sin (n \alpha T)
$$

Stiffness modulation will be affected by changing $\beta$, representing a gain factor for stiffness.

The spin-up profile for the class of problems considered is:

$$
\omega=\left\{\begin{array}{lc}
r t & 0<t<t_{o} \\
r t_{o} & t>t_{o}
\end{array}\right.
$$

This spin-up profile causes the differential equation of motion to contain coefficients are explicit functions of time, and the forcing sinusoid to be a function of $t^{2}$. The ramping 
nature of the forcing function contains a narrow band of frequencies about the instantaneous frequency of $n r t$. This results in the system's damped natural frequency being excited prior to the intersection of the instantaneous excitation frequency and the system's damped natural frequency.

\section{Smart Material Application -- Stiffness Scheduling}

Stiffness scheduling is defined as adapting the stiffness of the structure to reduce disturbance sensitivity. (This approach has been investigated in Reference 7 and Reference 8 , in which the stiffnesses at the supports of rotors are modulated.) Since the smart material described above can take on two distinct elastic moduli, the system will have two distinct damped natural frequencies. If the frequency responses for these two systems can be sufficiently separated, then the frequency response of the smart system can be greatly reduced.

Here we consider a system with the following dimensionless parameters:

$$
\begin{array}{rlr}
\xi & =0.02 & \\
n & =2 & \\
\tilde{A} & =0.1 & \\
\alpha & =\left\{\begin{array}{cc}
r T & 0<T<200.0 \\
200 r & T>200.0
\end{array}\right. \\
r & =0.0005 &
\end{array}
$$

A fourth order Runge-Kutta method was used to integrate the governing equation for this system for three cases:

- the dimensionless stiffness is set to a higher value;

- the dimensionless stiffness $\beta$ is set to a relatively low value;

- and dimensionless stiffness is appropriately switched during the ramp up of spin.

\subsection{Results}

Figure 6 shows the dimensionless tilt as a function of dimensionless time for the case where the torsional spring is held in a stiffer mode throughout: $\beta=4$. For that case, a large resonance behavior is seen near a dimensionless time of 120 . A beating occurs for a while after the shaft rew hes its terminal speed. More significantly, a fairly large oscillation remains at long times, after the disk has reached terminal speed.

Figure 7 shows the corresponding curve for the case of $\beta=1$. As expected, the resonancelike behavior occurs earlier in the process, when the excitation frequencies are lower. It is important that the behavior at long times manifests much lower amplitudes than is the case 


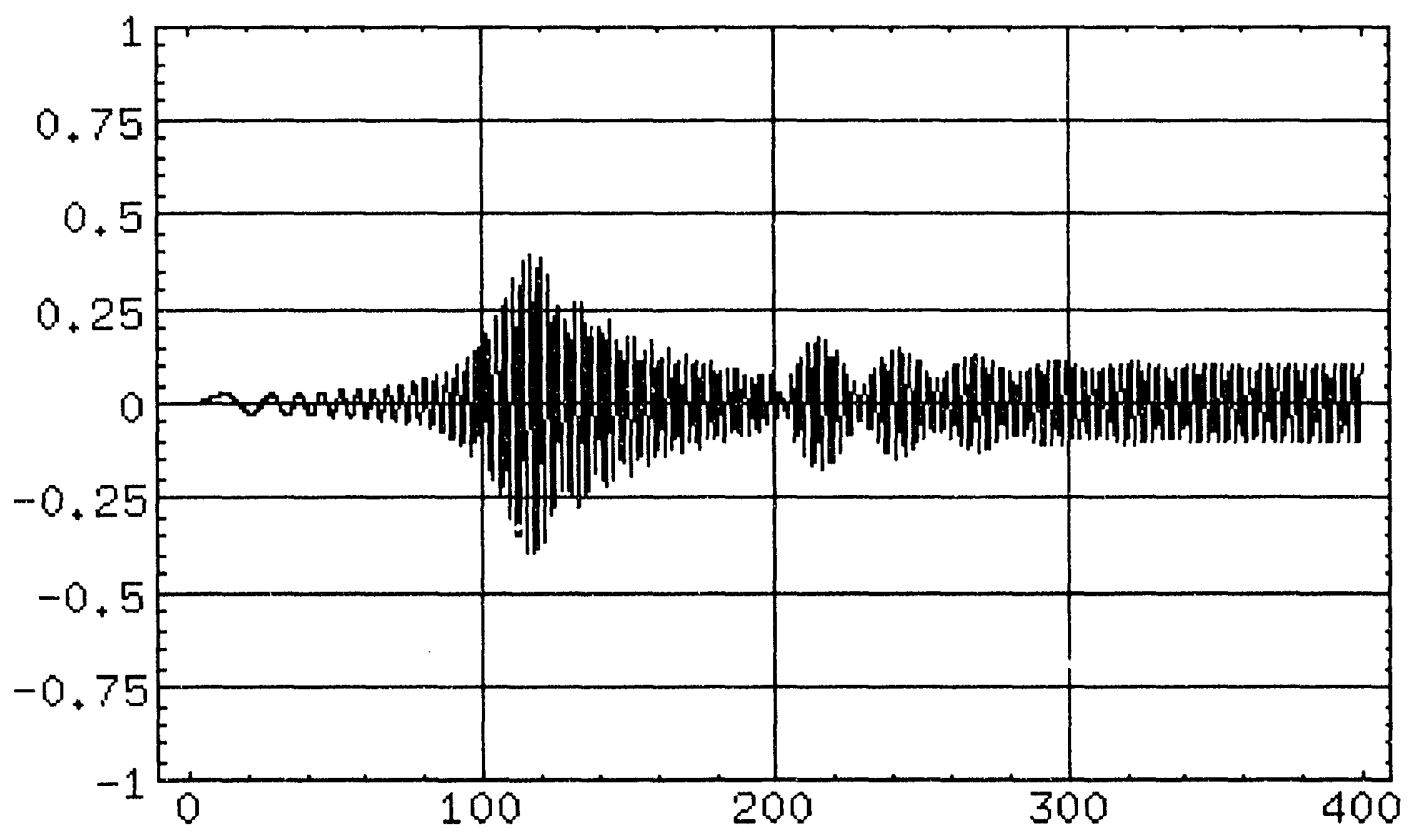

FI(YURE 6. Stiffening Actuator Turned On Throughout Spin-Up.

with the stiffer spring. This difference occures because the natural frequency in this case is further from the forcing frequency.

Figure 8 shows the dimensionless tilt as a function of dimensionless time where the stiffness parameter, $\beta$, is initially held at a value of 1.0 and instantaneously changed to a value of 4.0 at dimensionless time 98.0.

Note that the maximum amplitude in the resonance regime is slightly more than halved and that the long term oscillations are substantially lower in amplitude than those of the stiff system. Of course, this high frequency response is due to the stiffness of the structure at long times being that of the material shown in Figure 7, and the forced response at those frequencies are similar.

\subsection{Conclusions}

The smart material described here can be used in conjunction with stiffness scheduling to give a reduction in vibration amplitude for a class of rotating systems where the excitation is proportional to the spin rate. The time at which to change stiffness is based on the spinup profile being known in advance. This permits the best switching time to be chosen based on the frequency components of the excitation and the stiffness switching transients. 


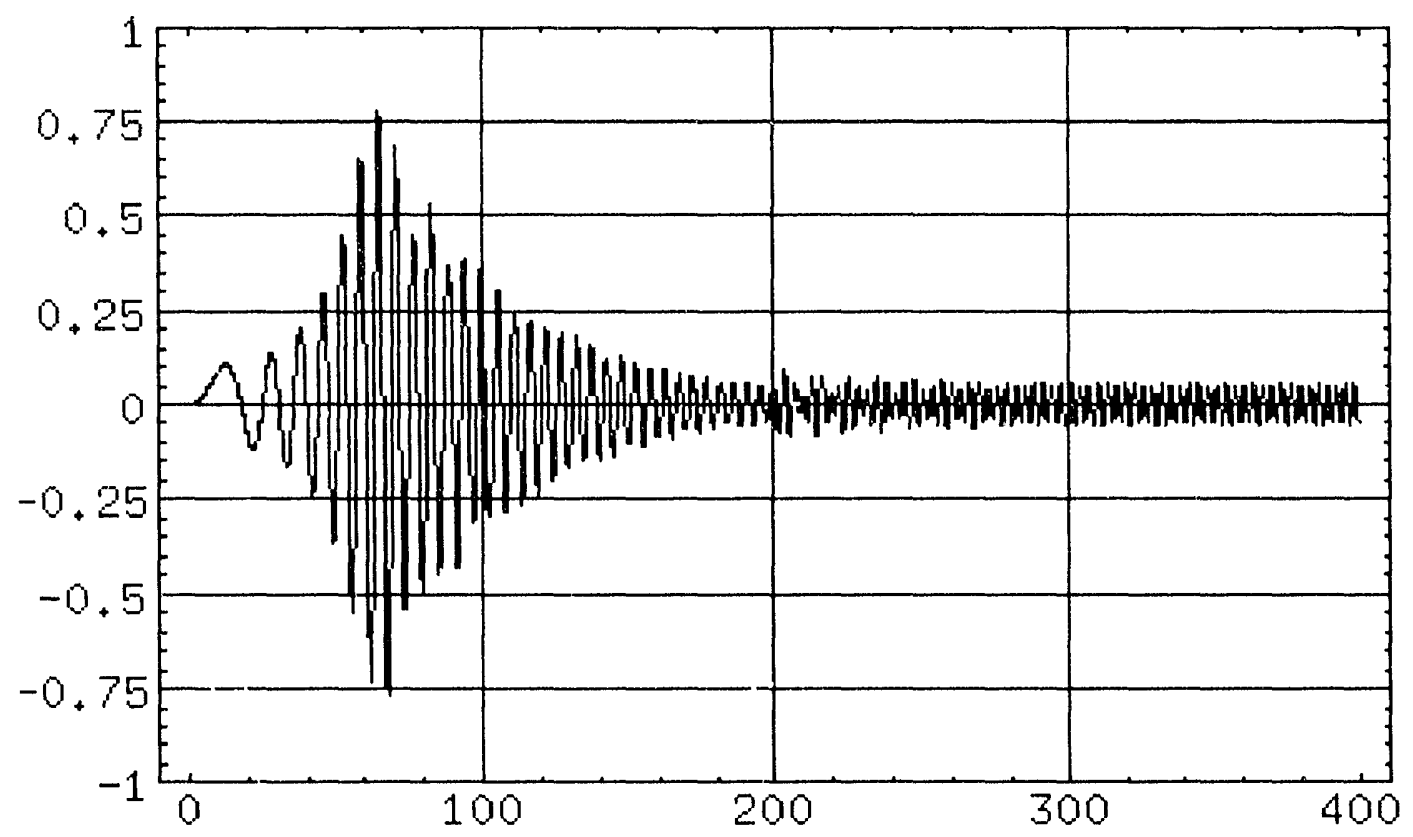

FICURL 7. Stiffening Actuator Turned (Off Throughout Spin

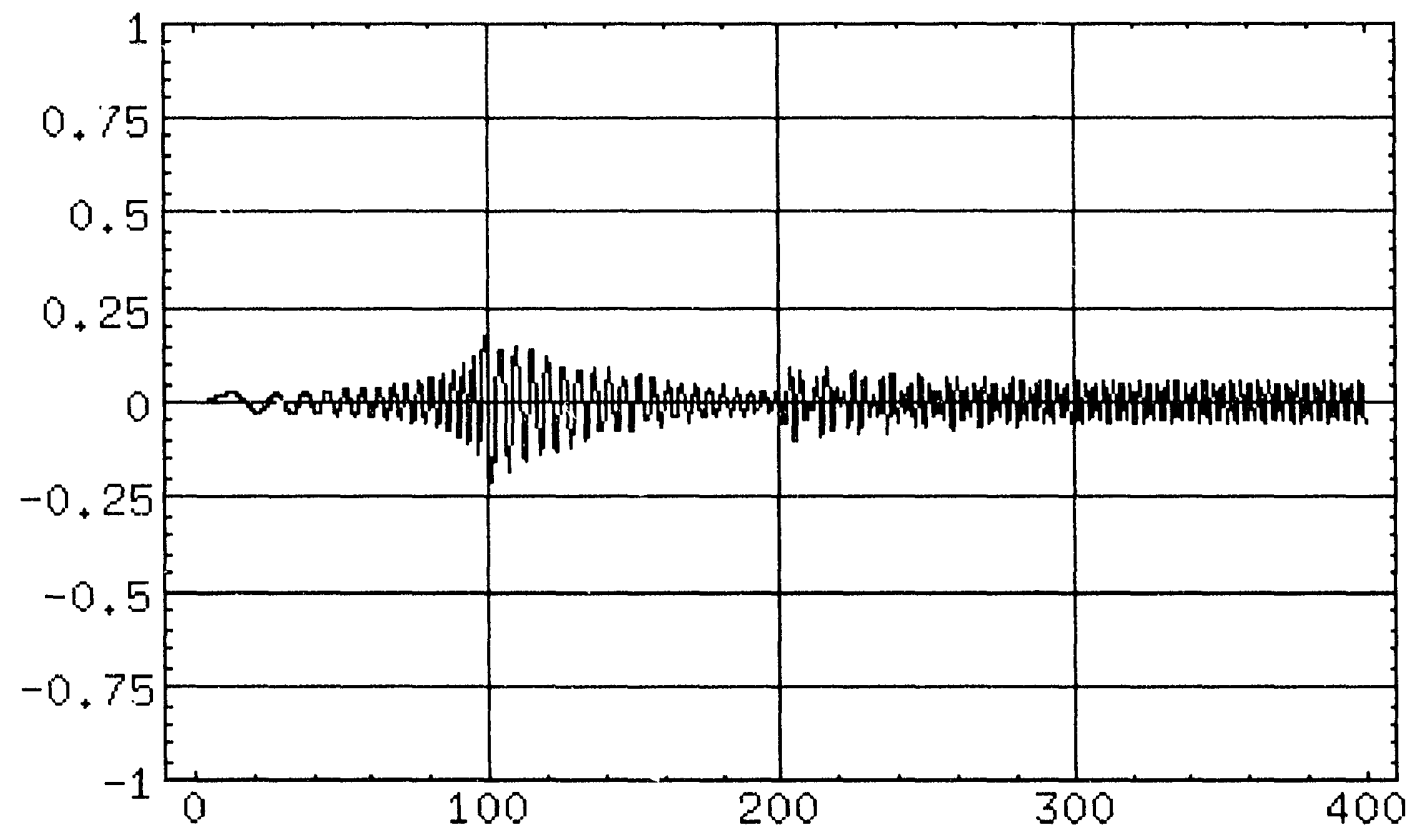

FIGURE 8. Amplitude Response with Stiffness Scheduling.

\subsection{Future Work}


A control law for choosing the stiffness switching time is being developed which does not require exact knowledge of the spin-up profile. Further, basic work in the materials issues of such actuators must be done.

\title{
DISCLAIMER
}

\begin{abstract}
This report was prepared as an account of work sponsored by an agency of the United States Government. Neither the United States Government nor any agency thereof, nor any of their employees, makes any warranty, express or implied, or assumes any legal liability or responsibility for the accuracy, completeness, or usefulness of any information, apparatus, product, or process disclosed, or represents that its use would not infringe privately owned rights. Reference herein to any specific commercial product, process, or service by trade name, trademark, manufacturer, or otherwise does not necessarily constitute or imply its endorsement, recommendation, or favoring by the United States Government or any agency thereof. The views and opinions of authors expressed herein do not necessarily state or reflect those of the United States Government or any agency thereof.
\end{abstract}

\subsection{References}

I. R. M. Christensen, Mechanics of Composite Materials, Krieger Publishing Company, Malabar, Florida, 1991.

2. Mike Zerkus and Jeff Akers, "Thermoelectrically controlled shape memory alloy actuators", Proceeciings of the Conference on Recent Advances in Adaptive and Sensory Materials and their Applications, held at Virginia Polytechnic Institute and State University, Blacksburg, Virginia, April 27-29, 1992.

3. A. Baz, S. Poh, J. Ro, M. Mutua, J. Gilheany, "Active control of Nitonol-Reinforced Composite beam”, Intelligent Structural Systems, H.S. Tsou and G. L. Anderson editors, Kluwer Academic Publishers, the Netherlands, 1992, pp 169-212.

4. Dimitris C. Lagoudas and Iradj G. Tadjbakhsh, "Active Flexible rods with embedded SMA fibers", Smart Materials and Structures 1, 1992, pp 162-167.

5. Jen-San Chen and D. B. Bogy, "Mathematical Structure of Modal Interactions in a Spinning Disk-Stationary Load System”, Journal of Applied Mechanics, Transactions of the ASME, Vol 59, June 1992, pp 39()-397

6. Daniel J. Segalman and Clark R. Dohrmann, 'Dynamics of Rotating Flexible Structures by a Method of Quadratic Modes', Sandia Report: SAND9()-2737, printed December 199()

7. Z. Viderman and I. Porat, "An optimal Control Method for Passage of a Flexible Rotor" Through Resonances", Journal of Dynamic Systems Measurement and Control, 109, 1987, pp 216-223

8. K. Nagaya, S. Takeda, Y. Psukui, 'T. Kumaido, "Active Control Method for passing through critical speeds of rotating shafts by changing stiffness of the supports with use of memory metals", Journal of Sound and Vibration, 113, 1987, pp 307-315. 

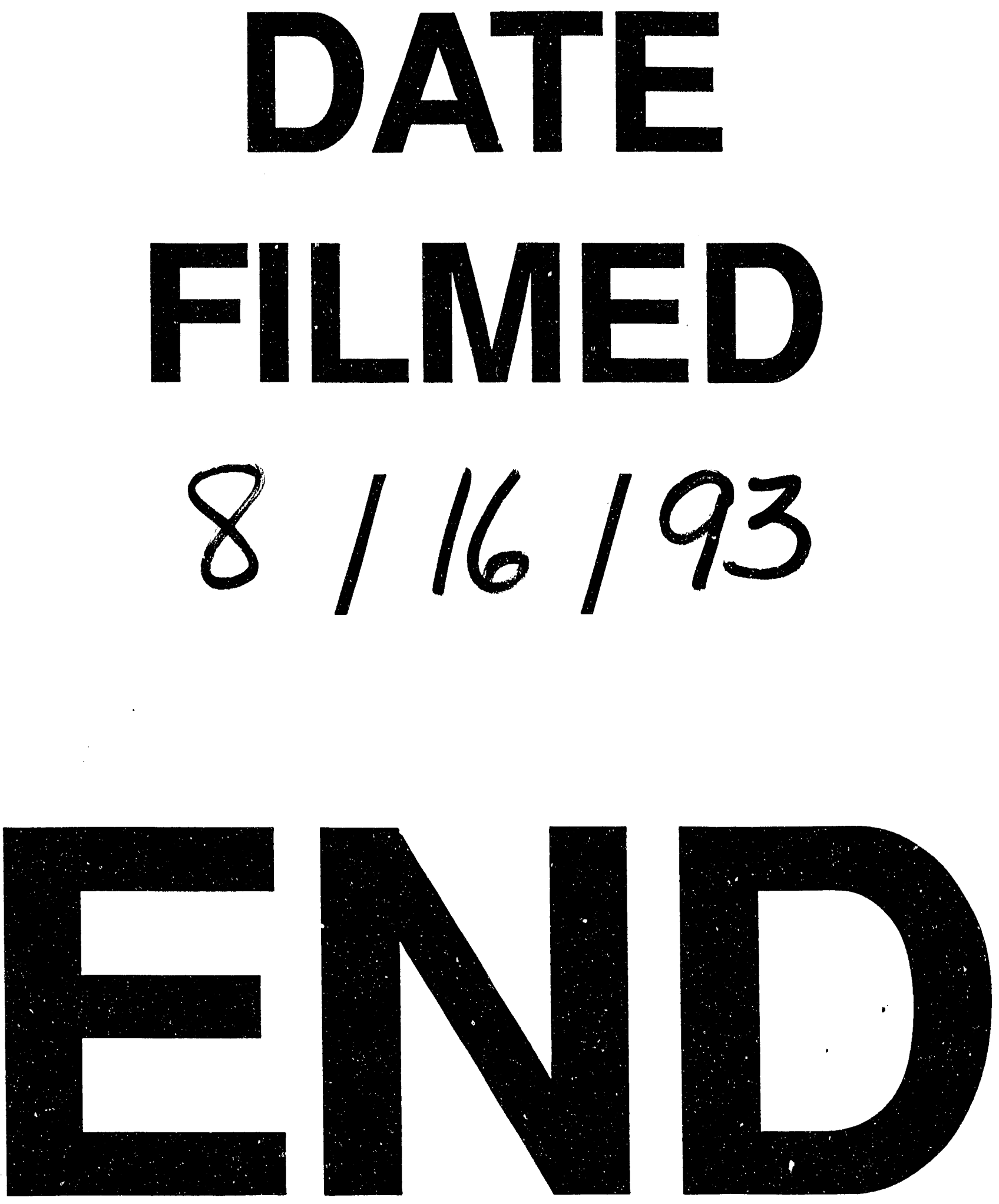